\title{
Inseguridad laboral y estrés percibido durante la Pandemia de COVID-19 en trabajadores de una corporación privada peruana
}

\author{
Job Insecurity and Stress During the COVID-19 Pandemic \\ in Workers at Private Peruvian Corporation \\ Elizabeth Ayacho Palma \\ Universidad Peruana Unión, Juliaca, Perú \\ (iD https://orcid.org/oooo-0oo2-8079-380X \\ Correspondencia: jetzad19@gmail.com \\ Oscar Javier Mamani-Benito \\ Universidad Peruana Unión, Juliaca, Perú \\ (iD https://orcid.org/oooo-ooo2-9818-2601 \\ Correo electrónico: oscar.mb@upeu.edu.pe
}

\section{Resumen}

La presente investigación tiene como objetivo determinar si la inseguridad laboral influye en la aparición de estrés percibido durante la pandemia de COVID-19 en trabajadores de una corporación privada en Perú. Corresponde a una investigación de diseño explicativo de corte transversal, y se contó con la participación de 205 trabajadores de una corporación privada peruana1 (84.4\% varones y 15.6\% mujeres), cuyas edades fluctúan entre 19 y 63 años. Los instrumentos utilizados para la recolección de información fueron la Escala de Inseguridad Laboral (LABOR-PE-COVID-19) y la Escala de Estrés Percibido relacionado con la pandemia COVID-19 (EEP-10-C). Los resultados obtenidos muestran que existe una relación directa y significativa entre las variables de estudio ( $r=.423 ; p<.05)$, de magnitud moderada. Aunado esto, el modelo de regresión lineal indica que la inseguridad laboral explica en un 17.5\% la varianza de la variable estrés percibido. Se concluye que la inseguridad laboral es un factor asociado al incremento de estrés en los trabajadores de una corporación privada del Perú. Esto puede tener repercusiones en la salud ocupacional y mental de los trabajadores, lo cual puede llevar a generar déficits en el rendimiento y producción es la empresa.

Palabras clave: Inseguridad laboral, estrés percibido, salud mental, salud ocupacional, COVID-19. 


\begin{abstract}
The present investigation aims to determine if insecurity at work influences the appearance of perceived stress during the COVID-19 pandemic in workers at a private corporation in Peru. It corresponds to an explanatory design investigation of a cross-sectional nature, with the participation of 205 workers at a private Peruvian corporation (84.4\% men and $15.6 \%$ women), whose ages fluctuate between 19 and 63 years. The instruments used for data collection were the Labor Insecurity Scale (LABOR-PE-COVID-19) and the Perceived Stress Scale related to the COVID-19 Pandemic (EEP-10-C). The results obtained show that there was a direct and significant relationship between the study variables ( $r=$ .423; $p<.05$ ), of moderate magnitude. In addition, the linear regression model indicated that job insecurity explained $17.5 \%$ of the variance of the perceived stress variable. It is concluded that job insecurity is a factor associated with increased stress in the workers at a private corporation in Peru. This can have repercussions on the occupational and mental health of the workers, which can lead to deficits in the company's performance and production.

Keywords: Job insecurity, perceived stress, mental health, occupational health, COVID-19.
\end{abstract}

\section{Introducción}

Percibir inseguridad laboral es una fuente de estrés para muchos trabajadores, esto, por las tasas elevadas de desempleo durante el periodo de crisis económica a causa de la pandemia por la COVID-19 (OIT, 2020a). En tal sentido, se puede definir la inseguridad laboral como la incapacidad de percibir y mantener la continuidad laboral frente a una situación de amenaza en el trabajo (Sora et al., 2014).

La Organización Internacional de Trabajo (OIT, 2020a) menciona que, a causa de la emergencia sanitaria, en varios países se ha optado por cerrar los centros de operaciones laborales. En Latinoamérica, la crisis de la pandemia ha dejado a más de 144 millones de trabajadores informales en una situación de vulnerabilidad y desprotección, quienes se ven obligados a seguir buscando trabajo para tener ingresos, aunque sea mínimos. Aunado a esto, la misma OIT (202ob) menciona que esta crisis también está produciendo problemas de salud mental como depresión, estrés, temor a perder empleo, e inseguridad respecto al futuro; y por otro lado, miedo al contagio y agotamiento emocional (Lozano-Vargas, 2020).

Esta situación en cifras, revela que en países de Latinoamérica como Colombia, se tiene un $41.8 \%$ de trabajadores que se encuentran en sectores de mayor riesgo, le siguen, Argentina con un 41.8\%, Chile, Ecuador, Honduras y Nicaragua con casi un $40 \%$, y Perú con poco más del $40 \%$ (Orgaz, 2020). En cuanto a este último, a consecuencia de la pandemia se estima una pérdida de aproximadamente 3.5 millones de empleos (Vinelli \& Murer, 2020). 
En función de lo encontrado en la literatura científica, los resultados de investigaciones acerca del impacto de la COVID-19 sobre la fuerza laboral recién vienen apareciendo. Aunque en algunos contextos como Alemania, Reichert y Tauchmann (2017) dan cuenta de que el ámbito laboral estaba siendo afectado incluso antes de la pandemia por COVID-19; y en el escenario actual, Xafis (2020) resalta el hecho de que la emergencia sanitaria viene repercutiendo negativamente en el ámbito laboral, por lo que existe la necesidad de que las personas reinventen sus actividades laborales y se contextualicen hacia las necesidades actuales. Así también, Ozamiz et al. (2020) revelaron la existencia de estrés, ansiedad y depresión en la fase de brote de la COVID-19, que fueron más altas en jóvenes. Por otra parte, en China, Lozano (2020) detectó que un $35 \%$ de la población en general sufría de distrés psicológico, sobre todo en grupos etarios con edades entre 18 a 30 años, donde las mujeres tenían mayores niveles que los hombres.

Ante esto, los autores de la presente investigación asumen la hipótesis de que percibir inseguridad laboral afecta la salud mental de las personas laboralmente activas, generando en ellos estrés a causa de la incertidumbre por el futuro. Por lo tanto, el objetivo de la presente investigación es determinar si la inseguridad laboral influye en la aparición de estrés percibido durante la pandemia por la COVID-19 en trabajadores de una corporación privada en Perú.

\section{Método}

\section{Diseño}

Estudio explicativo de corte transversal (Hernández et al., 2014).

\section{Participantes}

Se trabajó con una población de 205 trabajadores de ambos sexos, 173 hombres (84.4\%) y 32 mujeres (15.6\%), cuyas edades fluctúan entre 19 y 63 años. Estos pertenecen a la Corporación Andina de Gas Perú S.A.C. de las siguientes regiones: 48 de Arequipa (23.4\%), 33 de Cusco (16.1\%), 58 de Lima (28.3\%), 28 de Puno (13.7\%) y 38 de Tacna (13.7\%). En este caso, fueron seleccionados mediante un muestreo no probabilístico intencional con criterios de inclusión y exclusión.

\section{Instrumentos}

Escala de Inseguridad Laboral Percibida (LABOR-PE-COVID-19) validado al Perú por Mamani-Benito et al. (2020). Es un instrumento breve que tiene el objetivo de evaluar la percepción de inseguridad acerca de la condición laboral, el cual está compuesto por 4 ítems distribuidos en un solo factor. Las opciones de respuesta son de tipo Lickert con 5 categorías, desde "Totalmente en desacuerdo", hasta "Totalmente de acuerdo". En cuanto a su validez, demuestra un coeficiente $V$ de Aiken de .96, producto de la evaluación de 7 jueces expertos (psicólogos organizacionales) y la confiabilidad de la EILP en el presente estudio, fue $\alpha=.93$ (IC95\%= $.91-.94)$. 
Escala de Estrés Percibido relacionado con la Pandemia de COVID-19 (EEP-10-C; Campo-Arias et al. 2020). Es un instrumento breve que tiene el objetivo de evaluar el nivel de estrés que perciben los sujetos ante cualquier circunstancia de su entornoy personal. Consta de 10 ítems distribuidos en dos factores, que tiene opciones de respuesta tipo Lickert con 5 categorías que van desde "Nunca" hasta "Muy a menudo". En cuanto a su validez, demuestra un coeficiente $V$ de Aiken de .97, producto de la evaluación de 7 jueces expertos (psicólogos organizacionales) y la confiabilidad de la EEP-10-C en el presente estudio, fue $\alpha=.89$ (IC95\%: .85 - .92).

\section{Procedimientos}

Debido a la emergencia sanitaria se elaboró un formulario en Google Forms, el cual estuvo disponible en línea desde el 14 de junio hasta el 23 de julio del 2020. En la primera parte se puso el consentimiento informado donde, los participantes podían visualizar el objetivo y los fines de la investigación; así mismo, se detalló que la información brindada iba a tener un manejo estrictamente confidencial. La estrategia para la encuesta implicó compartir el link del formulario a través de correos electrónicos de los trabajadores de la empresa y redes sociales como Facebook y WhatsApp.

\section{Consideraciones éticas}

La investigación fue aprobada por el comité de ética de la Corporación Andina de Gas Perú S.A.C.

\section{Análisis de datos}

Primero, se realizó un análisis descriptivo de las variables: inseguridad laboral y estrés percibido. Segundo, se analizó las diferencias de las variables según el sexo, para lo cual, se utilizó el estadístico $t$ de Student y la $d$ de Cohen como medida del tamaño del efecto (TE) para la comparación de dos grupos independientes, donde valores de $.20, .50$ y .80 expresan un TE pequeño, moderado y grande. El análisis correlacional se llevó a cabo con el coeficiente de Pearson $(r \geq .50=$ grande, $r \geq .30=$ mediana $y r \geq$ $.10=$ pequeña). Por último, se estimó un modelo de regresión, calculando el TE en función del coeficiente de determinación ( $\left.R_{2}\right)$ y sus intervalos de confianza, donde valores $\geq 0,02, \geq 0,13 y \geq 0,26$ indican una ME pequeña, promedio y grande, respectivamente.

\section{Resultados}

En cuanto a las características demográficas de los participantes, se observa una mayor participación de varones (84.4\%) que mujeres (15.6\%). Así también, el rango de edad con más frecuencia es la de 19 a 25 años (38\%) seguido por la de 31 a 40 años (30.7\%). En la misma línea, las sedes con mayor proporción de participantes fueron la de Lima $(28.3 \%)$ y Arequipa (23.4\%). Por otra parte, en cuanto a la profesión con mayor porcentaje de participantes se ubica la de envasador (10.7\%). Finalmente, el 41.5\% vienen trabajando en la empresa por dos años (ver Tabla 1). 
Tabla 1. Características de los participantes

\begin{tabular}{|c|c|c|c|}
\hline Variable & Categoría & Frecuencia & Porcentaje \\
\hline \multirow{4}{*}{ Sexo } & Varón & 173 & 84.4 \\
\hline & Mujer & 32 & 15.6 \\
\hline & 19 a 25 años & 78 & 38.0 \\
\hline & 26 a 30 años & 31 & 15.1 \\
\hline \multirow[t]{5}{*}{ Edad } & 31 a 40 años & 63 & 30.7 \\
\hline & 41 a 50 años & 30 & 14.6 \\
\hline & Más de 51 años & 3 & 1.5 \\
\hline & Arequipa & 48 & 23.4 \\
\hline & Cusco & 33 & 16.1 \\
\hline \multirow[t]{13}{*}{ Lugar donde trabaja } & Lima & $\begin{array}{l}33 \\
58\end{array}$ & 28.3 \\
\hline & Puno & 28 & 13.7 \\
\hline & Tacna & 38 & 18.5 \\
\hline & Abogado & 5 & 2.4 \\
\hline & Administrador & 6 & 2.9 \\
\hline & Analista de créditos & 7 & 3.4 \\
\hline & Asistente & 11 & 5.4 \\
\hline & Ayudante de camión & 20 & 9.8 \\
\hline & Ayudante de carga & 18 & 8.8 \\
\hline & Cobrador & 20 & 9.8 \\
\hline & Conductor & 20 & 9.8 \\
\hline & Contador & 7 & 3.4 \\
\hline & Economista & 4 & 2.0 \\
\hline \multirow{14}{*}{ Puesto laboral } & Envasador & 22 & 10.7 \\
\hline & Gerente & 1 & .5 \\
\hline & Gerente regional & 6 & 2.9 \\
\hline & Ingeniero químico & 6 & 2.9 \\
\hline & Ingeniero de hidrocarburo & 11 & $5 \cdot 4$ \\
\hline & Jefe de patio & 7 & 3.4 \\
\hline & Jefe de venta & 5 & 2.4 \\
\hline & Operario & 6 & 2.9 \\
\hline & Psicólogo & 8 & 3.9 \\
\hline & Secretaria & 7 & 3.4 \\
\hline & Seguridad & 5 & 2.4 \\
\hline & Supervisor de ventas & 3 & 1.5 \\
\hline & 1 año & 38 & 18.5 \\
\hline & 2 años & 85 & 41.5 \\
\hline \multirow[t]{2}{*}{ Años en la empresa } & 3 años & 55 & 26.8 \\
\hline & 4 años a más & 27 & 13.2 \\
\hline
\end{tabular}

La Tabla 2 da cuenta, según la prueba $t$, que los varones presentan mayor inseguque no existe diferencia significativa en ridad laboral y estrés percibido. Respecto la inseguridad laboral $(\mathrm{t}=1.515 ; \mathrm{p}=.131) \quad$ al tamaño del efecto calculado mediante entre varones y mujeres. En cambio, si el d de Cohen, se evidencia que la variable existe diferencia significativa en relación inseguridad laboral se encuentra dentro al estrés percibido $(\mathrm{t}=184 ; \mathrm{p}=.854)$. Al del límite $(\mathrm{d}=.28)$ y para la variable estrés analizar los valores de la media se observa percibido es insignificante $(d=.03)$. 
Tabla 2. Inseguridad Laboral y Estrés Percibido entre varones y mujeres

\begin{tabular}{|lccccccc|}
\hline \multirow{2}{*}{ Variables } & \multicolumn{2}{c}{ Varones } & \multicolumn{2}{c}{ Mujeres } & \multirow{2}{*}{. } & \multirow{2}{*}{$\mathbf{~}$} \\
& $\mathbf{M}$ & DS & $\mathbf{M}$ & DS & & & \\
\hline Inseguridad laboral & 12.02 & 2.94 & 11.15 & 3.24 & 1.515 & .131 & .28 \\
Estrés percibido & 21.04 & 4.87 & 20.87 & 4.60 & .184 & .854 & .03 \\
\hline
\end{tabular}

En la Tabla 3 se observan las correlaciones Esto quiere decir que a mayor inseguridad entre las variables de estudio, así, se eviden- laboral existe mayor probabilidad de expecia una correlación directa y significativa rimentar estrés laboral en los participantes $(\mathrm{r}=.423, \mathrm{p}<.05)$ de magnitud moderada. de una corporación privada peruana.

Tabla 3. Análisis de correlaciones

\begin{tabular}{|llrr|}
\hline & & Inseguridad laboral & Estrés percibido \\
\hline Inseguridad & Correlación de Pearson & 1 & $.423^{* *}$ \\
laboral & Sig. (bilateral) & & .000 \\
& $\mathrm{~N}$ & 205 & 205 \\
Estrés percibido & Correlación de Pearson & $.423^{* *}$ & 1 \\
& Sig. (bilateral) & .000 & 205 \\
& $\mathrm{~N}$ & 205 & \\
\end{tabular}

Nota: ${ }^{* *}$ La correlación es significativa en el nivel o,o1 ( 2 colas).

Para determinar si la inseguridad laboral influye sobre el estrés percibido, se realizó un análisis de regresión lineal. En tal sentido, la Tabla 4 evidencia los coeficientes de correlación múltiple $\mathrm{R}, \mathrm{R}^{2}, \mathrm{R}^{2}$ corregida, error estándar de la estimación (EE), y el valor F de ANOVA.

Se observa que el coeficiente de determinación $\mathrm{R}^{2}=.179$ indica que la variable inseguridad laboral explica el 17,9\% de la varianza total de la variable Estrés Percibido. Un valor mayor del coeficiente de determinación múltiple, indica un mejor poder explicativo de la ecuación regresión, y por lo tanto, mayor poder de predicción de la variable dependiente. La $\mathrm{R}^{2}$ corregida, explica el $17.5 \%$. El valor $\mathrm{F}$ de ANOVA $(\mathrm{F}=44,290 ; \mathrm{p}=.000)$ indican que existe una relación lineal significativa entre la variable inseguridad laboral (predictora) y la variable estrés percibido (criterio). 
Tabla 4. Coeficientes de correlación lineal $R, R 2, R 2$ corregida, EE, $F$

\begin{tabular}{|ccccccc|}
\hline Modelo & $\mathbf{R}$ & $\mathbf{R}^{2}$ & $\mathbf{R}^{2}$ corregida & EE & $\mathbf{F}$ & Sig \\
\hline 1 & .423 & .179 & .175 & 4.377 & 44.290 & .000 \\
\hline
\end{tabular}

Nota: Variables predictoras $=($ Constante $)$ Inseguridad laboral; Variable dependiente $=$ Estrés percibido

En la Tabla 5, se muestra coeficientes de regresión no estandarizados (B), coeficientes de regresión estandarizados $(\beta)$ y los estadísticos relacionados con la variable predictora. El coeficiente $(.423)$ indica que la inseguridad laboral (variable predictora) predice significativamente el estrés percibido (variable criterio). El valor t de los coeficientes de regresión beta de la variable predictora esaltamentesignificativo $(\mathrm{p}<.01)$.

Tabla 5. Coeficientes de regresión lineal B (no estandarizados), $\beta$ (estandarizados) y test $t$

\begin{tabular}{|lccccc|}
\hline Modelo & B & EE & $\boldsymbol{\beta}$ & $\mathbf{t}$ & Sig \\
\hline (Constante) & 12.942 & 1.252 & & 10.341 & .000 \\
Inseguridad laboral & .679 & .102 & .423 & 6.655 & .000 \\
\hline
\end{tabular}

Nota: Variable dependiente $=$ Estrés percibido

\section{Discusión}

Las repercusiones a causa de la pandemia por COVID-19 han afectado no solo la salud de la población, sino también, el ámbito laboral y económico de las personas (Mejía et al., 2020). En tal sentido, miles de trabajadores peruanos, sobre todo del ámbito informal vienen experimentando niveles significativos de inseguridad laboral (Bohoslavsky \& Rulli, 2020); por otra parte, quienes ocupan puestos laborales en empresas no son ajenos a esta reacción, pues la emergencia sanitaria ha causado que muchas de las empresas se acojan a acciones como la suspensión perfecta de labores (Diario Gestión, 2020).

La presente investigación tuvo el objetivo de determinar si la inseguridad laboral influye en la aparición de estrés percibido en trabajadores peruanos. $\mathrm{Al}$ respecto, en investigaciones recientes ya se demostraba que los trabajadores peruanos percibían tener condiciones de trabajo, seguridad y salud deficientes (Sabastizagal-Vela et al., 2020). Precisamente, el principal hallazgo en la presente investigación tiene que ver con el hecho de que la inseguridad laboral es un factor que explica la aparición de estrés percibido en trabajadores peruanos, pues según el análisis de regresión la variable inseguridad explica el $17.5 \%$ de la varianza de la variable estrés percibido.

Este resultado confirma lo mencionado por la Organización Internacional del Trabajo (OIT, 2016) que desde hace algunos años informó que la escasez de 
trabajo y la inseguridad laboral son las principales causas del estrés en adultos laboralmente activos. Así mismo, en una investigación realizada en España por Sora, Caballer y Peiró (2014) se concluyó que la inseguridad laboral se ha convertido en una fuente de estrés para muchos trabajadores en tiempos de crisis económica, demostrando que esta variable es uno de los estresores más nocivos para los trabajadores laboralmente activos. Por último, el Centro para el Control y la Prevencion de Enfermedades Respiratorias (NCIRD, 2020) menciona que en el contexto actual ha cambiado la forma de trabajo de muchas personas, pues los trabajadores sienten preocupación por el riesgo de exposición al virus en el trabajo, a perder el trabajo, a cambios en su carga de trabajo, a tener que aprender nuevas herramientas de comunicación, y a la par, tener que atender necesidades personales, familiares y laborales.

Una explicación teórica acerca de estos hallazgos surge al reconocer que la inseguridad laboral y el miedo a perder el trabajo son considerados factores estresantes en la literatura científica, más fuertes incluso que la sobrecarga laboral (Peraza, 2020). Esto guarda coherencia al descubrir que el trabajo da sentido a la vida de muchas personas (Céspedes \& Martínez, 2016), por lo que la idea de no poder trabajar puede generar una sensación de fracaso personal, que a su vez puede ocasionar cambios en el carácter y las emociones (Coduti et al., 2013). Por otra parte, el hecho de que el trabajador se sienta inseguro en cuanto a su trabajo, es perjudicial para su salud mental y para la organización en la que labora (Parra-Tapia et al., 2019), puede ser un factor para la disminución del rendimiento laboral (Valencia \& Avendaño, 2014).

El estudio presenta algunas limitaciones, la primera, tiene que ver con la cantidad de participantes y su delimitación geográfica. En este caso, los resultados no pueden ser generalizados para todos los trabajadores peruanos, sino, para los de la corporación privada peruana en la que se realizó el estudio; por lo tanto, futuras investigaciones deberían ampliar el espectro de la población e incluir sobre todo el área informal. En segundo lugar, el tipo de muestreo, que no permitió una selección aleatoria de la muestra.

Se concluye que la inseguridad laboral influye sobre el estrés percibido en trabajadores de una corporación privada peruana. Esto puede tener repercusiones en la salud ocupacional y mental de los trabajadores, lo cual puede llevar a generar déficit en el rendimiento y producción de la empresa privada.

\section{Financiamiento}

La presente investigación fue autofinanciada.

\section{Conflictos de interés}

Los autores declaran que no tienen conflictos de interés. 


\section{Referencias}

Bohoslavsky, J. P., \& Rulli, M. (2020). Covid-19, international financial institutions and the continuity of androcentric policies in Latin America. Revista Estudos Feministas, 28(2), 1-16. https://doi.org/10.159o/1806-9584-2020v28n273510

Campo-Arias, A., Pedrozo-Cortés, M. J., \& Pedrozo-Pupo, J. C. (2020). Escala de estrés percibido relacionado con la pandemia de COVID-19: una exploración del desempeño psicométrico en línea. Revista Colombiana de Psiquiatria, 49(4), 229-230. https://doi.org/10.1016/j.rcp.2020.05.005

Cespedes, G., \& Martínez, J. (2016). Un análisis de la seguridad y salud en el trabajo en el sistema empresarial cubano. Revista Latinoamericana de Derecho Social, 22. http:// www.scielo.org.mx/scielo.php?script=sci_arttext\&pid=S1870-46702016000100001

Coduti, P. S., Gattás, Y. B., Sarmiento, S. L., \& Schmid, R. A. (2013). Enfermedades Laborales: cómo afectan el entorno organizacional. (Tesis de grado). Universidad Nacional de Cuyo, Mendoza, Argentina. https://bdigital.uncu.edu.ar/objetos_digitales/5215/codutitesisenfermedadeslaborales.pdf

Diario Gestión. (2020). MTPE sobre suspensión perfecta: de 30, ooo solicitudes solo se han aprobado 1,0oo. https://gestion.pe/peru/coronavirus-peru-ministra-de-trabajo-sobre-suspension-perfecta-de-30-mil-solicitudes-solo-se-han-procesado-mil-covid-19-nndc-noticia/

Hernández, R., Fernández, R., \& Baptista, P. (2014). Metodologia de la Investigación. McGraw-Hill.

Lozano-Vargas, A. (2020). Impacto de la epidemia del Coronavirus (COVID-19) en la salud mental del personal de salud y en la población general de China. Revista de Neuro-Psiquiatria, 83(1), 51-56. https://doi.org/10.20453/rnp.v83i1.3687

Mamani-Benito, O., Apaza, E., Carranza, R., Rodriguez-Alarcon, J. F., \& Mejia, C. (2020). Inseguridad laboral en el empleo percibida ante el impacto del COVID-19 : validación de un instrumento en trabajadores peruanos. Revista de la Asociación Española de Medicina y Trabajo, 29(3), 184-185. http://scielo.isciii.es/pdf/medtra/ v29n3/1132-6255-medtra-29-03-184.pdf

Mejia, C., Rodriguez-Alarcon, F., Garay-Rios, L., Enrique-Anco, M., Moreno, A., Huaytan-Rojas, K., Huancahuari-Nañac, N., Julca-Gonzales, A., Alvarez, C., Choque-Vargas, J., \& Curioso, W. (2020). Percepción de miedo o exageración que 
transmiten los medios de comunicación en la población peruana durante la pandemia de la COVID-19. Revista Cubana de Investigaciones Biomedicas, 39(2), e698. http://scielo.sld.cu/scielo.php?script=sci_arttext\&pid=So864-03002020000200001

NCIRD (2020). Centro para el control y la prevencion de Enfermedades Respiratorias. https://espanol.cdc.gov/ncird/div/flu/service-america-medal.html

Organizacion Internacional del Trabajo (2016). Estres laboral. https://www.ilo.org/ wcmsp5/groups/public/---americas/---ro-lima/documents/genericdocument/ wcms_475146.pdf

Organización Internacional del Trabajo. (2020a). Frente a la pandemia: Garantizar la Seguridad y Salud en el Trabajo. https://www.ilo.org/wcmsp5/groups/public/--ed_protect/---protrav/---safework/documents/publication/wcms_742732.pdf

Organización Internacional del Trabajo. (2020b). El COVID-19 y el mundo del trabajo. Segunda edición Estimaciones actualizadas y análisis. https://www.ilo.org/wcmsp5/ groups/public/---dgreports/---dcomm/documents/briefingnote/wcms_740981.pdf

Orgaz, C. (2020). Coronavirus: Coronavirus: "Se perderán 195 millones de empleos en solo 3 meses" por la pandemia, el alarmante informe de la OIT (y cómo afectará a América Latina). https://www.bbc.com/mundo/noticias-america-latina-5222009o\#: :text=El\%2ocovid\%2D19\%2ohar\%C $3 \%{ }_{3} \%$ \%2odesaparecer,poco\%20 tiempo\%2C\%2odicen\%2olos\%2oexpertos

Ozamiz-Etxebarria, N., Dosil-Santamaria, M., Picaza-Gorrochategui, M., \& IdoiagaMondragon, N. (2020). Niveles de estrés, ansiedad y depresión en la primera fase del brote del COVID-19 en una muestra recogida en el norte de España. Cadernos de Saude Publica, 36(4), eoo054020. https://doi.org/10.1590/0102-311Xooo54020

Parra-Tapia, E., Perales-Ortiz, G., Quezada, A., \& Torres-Pereda, P. (2019). Salud y seguridad laboral: intervención educativa en trabajadores de limpieza en áreas de investigación. Salud Publica de Mexico, 61(5), 657-699. https://scielosp.org/ $\mathrm{pdf} / \mathrm{spm} / 2019 . v 61 \mathrm{in}_{5} / 657-669 / \mathrm{es}$

Peraza, C. X. (2020). Salud laboral frente a la pandemia del COVID19 en Ecuador. Medisur, 18(3), 507-511. http://scielo.sld.cu/scielo. php?script=sci_arttext\&pid=S1727-897X2020000300507 
Reichert, R., \& Tauchmann, H. (2017). Workforce reduction, subjective job insecurity, and mental health. Journal of Economic Behavior and Organization, 133, 187-212. https://doi.org/10.1016/j.jebo.2016.10.018

Sabastizagal-Vela, I. L., Astete-Cornejo, J., \& Benavides, F. G. (2020). Working, safety and health conditions in the economically active and employed population in urban areas of Peru. Revista Peruana de Medicina Experimental y Salud Publica, 37(1), 32-41. https://doi.org/10.17843/rpmesp.2020.371.4592

Salas, S. (2018). La inseguridad laboral en la poblacion asalariada. (Trabajo de grado). Universidad Autonoma de Barcelona, Barcelona, España. https://ddd.uab.cat/ pub/tesis/2018/hdl_10803_665796/ssn1de1.pdf

Sora, B., Caballer, A., \& Peiró, J. M. (2014). La inseguridad laboral y sus consecuencias en un contexto de crisis económica. Papeles Del Psicologo, 35(1), 15-21. http://www. papelesdelpsicologo.es/pdf/2317.pdf

Valencia Silva, L., \& Avendaño Bravo, C. (2014). Inseguridad laboral percibida y malestar psicológico: Evaluación de dos modelos predictivos en trabajadores suministrados de la Ciudad de Concepción, Chile. Ciencia E Trabajo, 16(49), 49-55. https://doi.org/10.4067/so718-24492014000100009

Vinelli M., \& Maurer A. (2020). Impacto del COVID-19 en el empleo en el Perú. https://www.esan.edu.pe/conexion/actualidad/2020/o4/24/ impacto-del-covid-19-en-el-empleo-en-el-peru/

Xafis, V. (2020). 'What is Inconvenient for You is Life-saving for Me': How Health Inequities are playing out during the COVID-19 Pandemic. Asian Bioethics Review, 12(2), 223-234. https://doi.org/10.1007/s41649-020-00119-1

Recibido: 15 de enero de 2021

Revisado: 20 de abril de 2021

Aceptado: 20 de julio de 2021 